\title{
First Nationwide Survey on the Resistance to First Line Anti-Tuberculosis Drugs in Ghana
}

\author{
Kennedy K. Addo 1*, Richard Owusu', Christian Bonsu', Kwaku Owusu-Darko1, \\ Samuel O. Addo1, Gloria I. Mensah ${ }^{1}$, Mercy J. Newman ${ }^{2}$, David Ofori-Adjei ${ }^{1}$, Frank A. Bonsu ${ }^{3}$ \\ ${ }^{1}$ Department of Bacteriology, Noguchi Memorial Institute for Medical Research, University of Ghana, Accra, Ghana \\ ${ }^{2}$ Department of Microbiology, School of Biomedical and Allied Health Sciences, University of Ghana, Accra, Ghana \\ ${ }^{3}$ National Tuberculosis Control Programme, Accra, Ghana \\ Email: ^kaddo@noguchi.ug.edu.gh
}

How to cite this paper: Addo, K.K., Owusu, R., Bonsu, C., Owusu-Darko, K., Addo, S.O., Mensah, G.I., Newman, M.J., Ofori-Adjei, D. and Bonsu, F.A. (2018) First Nationwide Survey on the Resistance to First Line Anti-Tuberculosis Drugs in Ghana. Journal of Tuberculosis Research, 6, 68-80.

https://doi.org/10.4236/jtr.2018.61007

Received: February 13, 2018

Accepted: March 19, 2018

Published: March 22, 2018

Copyright $\odot 2018$ by authors and Scientific Research Publishing Inc. This work is licensed under the Creative Commons Attribution International License (CC BY 4.0).

http://creativecommons.org/licenses/by/4.0/

(C) (i) Open Access

\begin{abstract}
Background/objective: A nationwide survey on the resistance to first line anti-tuberculosis (anti-TB) drugs was conducted in Ghana from 2007-2008 by Noguchi Memorial Institute for Medical Research in collaboration with the National Tuberculosis Control Programme. We aimed to characterize mycobacterial species causing pulmonary tuberculosis (PTB) and determine the resistance pattern to first line anti-TB drugs among newly diagnosed and previously treated PTB patients in Ghana. Methods: Two sputum samples from consented new smear positive PTB patients who had never been treated for TB or had been on anti-TB treatment for less than a month and patients who had been treated for TB previously for more than a month in selected diagnostic centres nationwide were collected for culture, identification and drug susceptibility test. Culture positive isolates were tested against streptomycin $(\mathrm{S})$, isoniazid $(\mathrm{H})$, rifampicin $(\mathrm{R})$ and ethambutol (E) using the simplified proportion method and line probe assay (LPA). The LPA was performed in mid-2017. Results: Among 410 samples, 345 positive cultures were obtained and identified as Mycobacterium tuberculosis complex (MTBC). Of the 345 isolates, 133 were further differentiated by GenoType $\mathrm{MTBC}^{\circ}$ as $M$. tuberculosis, 126 (94.7\%) and M. africanum 7 (5.3\%). The overall drug resistance patterns were as follows: $43 / 345$ (12.5\%), 6/345 (1.7\%), 9/345 (2.6\%) and 71/345 (20.6\%) were resistant to $H, R, E$ and S respectively and 5/345 (1.4\%) were multi-drug resistant (MDR). Conclusion: The results indicate high levels of resistance to $\mathrm{S}$ and $\mathrm{H}$ among new and previously treated $\mathrm{TB}$ patients. We recommend adequate surveillance systems including periodic national anti-TB drug resistance surveys.
\end{abstract}




\section{Keywords}

Tuberculosis, National Survey, Drug Resistance, Ghana

\section{Introduction}

Tuberculosis (TB) is a disease of great antiquity that continues to be a major public health problem worldwide. The available data on TB in Ghana indicate that the disease burden is high and TB remains an important cause of major disability and death in the country [1]. The incidence of TB per 100,000 population in Ghana has seen a fifteen year gradual decreasing trend from 214 in 2001 to 160 in 2015 [2]. However, it was observed from the Ghana national TB prevalence survey conducted in 2013 that TB prevalence rate (286 per 100,000 population) was about four times higher than the previous estimate prior to the survey (personal communication with the programme manager for the National $\mathrm{Tu}$ berculosis Control Programme (NTP), Ghana, unpublished data). A serious threat to TB control is the emergence of drug resistant strains which are difficult to treat by standard short course regimens. In Ghana, the NTP has over the years focused on ensuring an effective Directly Observed Treatment Short course (DOTS) as a strategy to prevent the development of drug resistant TB. This has worked to a large extent as failure rates for new smear positive TB cases have remained consistently below $2 \%$ since 2007 [3]. While poor quality anti-TB drug prescriptions have also virtually been eliminated in Ghana, some incidents of poor case management remain which may partly explain the emergence of drug resistance TB. Current estimate of drug resistant TB prevalence is at $2.6 \%$ among new TB cases and this corresponds with the treatment failure rate among new cases, which ranges from between $2.7 \%$ and $3.5 \%$ since 1997 [4]. The main cause of anti-TB drug resistance is the failure to ensure correct treatment of each patient with TB. This can be due to improper prescription of treatment regimens, inadequate or irregular drug supply, poor case holding, poor quality of drugs, and failure to ensure that patients adhere to the prescribed regimens. Anti-TB drug resistance is classified as either primary drug resistance (resistance among new cases) or acquired resistance (resistance among previously treated case). Drug resistance among new cases is the presence of a resistant strain of $M$. tuberculosis in newly diagnosed patient who has never received anti-TB drugs or has received them less than one month of treatment. Drug resistance among previously treated cases is resistance found in a patient who has previously received at least one month of anti-TB treatment. Surveillance of anti-TB drug resistance is an essential tool for monitoring the effectiveness of TB control programmes and improving national and global TB control efforts. However, there has been no national survey on anti-TB drug resistance among sputum smear positive TB cases in Ghana since the establishment of the NTP in 1994. Studies done in some health facilities in Ghana over the years reported various levels of 
resistance however, they were not of national dimension [5] [6]. Therefore to ascertain and establish anti-TB drug sensitivity patterns nationwide we conducted a national survey on resistance to first line anti-TB drugs from 2007 to 2008. The objectives of the survey were to monitor the prevalence of drug resistance TB in Ghana; characterize various mycobacterial species causing pulmonary TB (PTB); determine the pattern of resistance among newly diagnosed and previously treated smear positive PTB patients to first-line anti-TB drugs in Ghana.

\section{Materials and Methods}

\subsection{Study Design}

The study was cross-sectional for eligible registered PTB patients in accordance with the World Health Organization (WHO) guidelines on anti-TB drug resistance surveillance [7].

\subsection{Study Population}

Based on the list of all diagnostic centres in the country with the number of newly registered patients in the centres per year a cumulative population list was compiled from which 30 clusters were selected.

\subsection{Inclusion Criteria}

All consented registered sputum smear positive PTB patients in the selected diagnostic centres who had never been treated for TB or had been treated for a period less than one month and all patients who had been treated for TB before for a period more than one month. Children under the age of 15 years who met the admission criteria were also included to give an indication of recent transmission of drug resistant strains in the community.

\subsection{Exclusion Criteria}

Patients with severe haemoptysis as well as smear negative PTB patients. In addition, patients receiving drug treatment at the time of the study for more than one month.

\subsection{Sampling Strategy}

A defined intake period of 6 months identical for all the selected diagnostic centres was used to collect the samples from November 2007 to April 2008. Due to large number of eligible cases, sampling was done using weighted cluster sampling method-a cluster sampling proportionate to size [7]. This avoided the risk of drawing a sample that misses the large diagnostic centres.

\subsection{Patient Registration}

Each patient that met the inclusion criteria was assisted to fill an intake form and assigned a serial registration number with a prefix unique to the selected diagnos- 
tic centre. This resulted in easy identification of the centre in cases of resistant strain detection. A detailed structured questionnaire was used to collect information on the clinical history and basic demographic data of the patients. Other forms were designed to retrieve information of patient including smear results from diagnostic center and sputum shipment details.

\subsection{Sputum Collection and Transport}

Two sputum samples (one spot and an early morning) were obtained from each eligible TB patient and an equal volume of $1 \%$ Cetylpyridinium Chloride (CPC) was added to each one for homogenization of the mucous and organic debris as well as for decontamination on transit and to maintain the viability of the bacilli. Samples were then transported on ice within one week to the Bacteriology Department of Noguchi Memorial Institute for Medical Research (NMIMR), for microbiological and molecular investigations.

\subsection{Culture and Strain Identification}

A preliminary diagnosis using Ziehl-Neelseen (ZN) staining technique was used to detect acid fast bacilli (AFB) in samples collected by smear examination. Specimens were then centrifuged at $3000 \mathrm{~g}$ for $15 \mathrm{mins}$ and inoculated onto two pairs of Lowenstein-Jensen (LJ) media with one pair containing glycerol and the other containing $0.5 \%$ sodium pyruvate. Cultures were incubated at $37^{\circ} \mathrm{C}$ for four to eight weeks. Identification of isolates was based on acid fastness, colony morphology, pigment production, rate of growth onto p-nitrobenzoic acid (PNB $500 \mathrm{mg} / \mathrm{l}$ ).

\subsection{Phenotypic Drug Susceptibility Testing}

Drug susceptibility testing (DST) was carried out according to the proportion method as described by WHO [8]. First line anti-TB drugs: isoniazid (H), rifampicin (R), streptomycin (S) and ethambutol (E), were incorporated into Lowenstein-Jensen (LJ) media at the following concentrations; $0.2 \mu \mathrm{g} / \mathrm{ml}, 40 \mu \mathrm{g} / \mathrm{ml}, 4.0$ $\mu \mathrm{g} / \mathrm{ml}$ and $2.0 \mu \mathrm{g} / \mathrm{ml}$ respectively. One hundred microliters of cell suspensions of each Mycobacterium strain was inoculated onto an LJ medium slope and incubated at $37^{\circ} \mathrm{C}$ for four to six weeks. Culture readings were done on the $28^{\text {th }}$ and the $35^{\text {th }}$ days after inoculation. A standard $\mathrm{H}_{37} \mathrm{R}_{\mathrm{v}}$ strain and reference drug-resistant strains were used as positive controls while inoculated drug-free media were used as negative controls.

\subsection{Genotyping and Molecular Drug Susceptibility Testing}

One hundred and thirty-three (133) isolates originally identified and conventional DST done were subsequently confirmed between May and July 2017 as a quality assurance measure using line probe assay-the GenoType MTBC and GenoType MTBDRplus respectively. Both kits contain the same set of reagents (Denaturation, Hybridization, Stringent, Rinsing, Conjugate, and Substrate) and follow the same procedure for running the assay. However, they differ in the 
type of probes (oligonucleotides) on the test strip. The procedure involving DNA extraction, polymerase chain reaction (PCR) and reverse hybridization were done according to manufacturer's instructions [9] [10] as described briefly below.

\subsubsection{DNA Extraction}

DNA was extracted with GenoLyse. Briefly, $500 \mu \mathrm{l}$ of stored isolates was transferred into a $1.5-\mathrm{ml}$ screw cap tube and centrifuged for $15 \mathrm{~min}$ at $10,000 \times \mathrm{g}$ in a standard tabletop centrifuge. The pellet was resuspended in $100 \mu \mathrm{l}$ of an alcalic lysis (A-LYS) buffer and incubated for $5 \mathrm{~min}$ at $95^{\circ} \mathrm{C}$ in a heat block. Subsequently, $100 \mu \mathrm{l}$ of neutralization buffer was added to lysate, vortexed, and centrifuged for $5 \mathrm{~min}$ at full speed in a tabletop centrifuge. Finally, the supernatant containing the mycobacterial DNA was carefully transferred into different tubes using Pasteur pippette and kept at $-20^{\circ} \mathrm{C}$ until used for PCR.

\subsubsection{Multiplex Amplification with Biotinylated Primers}

Briefly, $10 \mu \mathrm{l}$ of AM-A (5 $\mu \mathrm{l} \mathrm{10x}$ buffer, $2 \mu \mathrm{l} \mathrm{MgCl}_{2}, 3 \mu \mathrm{l}$ of molecular grade water, $0.2 \mu \mathrm{l}(\mathrm{UU}) \mathrm{Taq}$ DNA polymerase) was mixed with $35 \mu \mathrm{l}$ of AM-B (nucleotides, biotinylated primers, dye). Then, $5 \mu \mathrm{l}$ of extracted DNA sample was added to the master-mix in another room. PCR amplification was done with the thermocycler set at the following cycling conditions: one cycle at $95^{\circ} \mathrm{C}$ for 15 minutes, followed by ten cycles at $95^{\circ} \mathrm{C}$ and $58^{\circ} \mathrm{C}$ for 30 seconds and two minutes respectively. This was followed by another round of 20 cycles at $95^{\circ} \mathrm{C}, 53^{\circ} \mathrm{C}$ and $70^{\circ} \mathrm{C}$ for 25,40 and 40 seconds respectively before a final single cycle at $70^{\circ} \mathrm{C}$ for eight minutes.

\subsubsection{Reverse Hybridization}

GT-Blot $48^{\circledR}$ automated hybridizer (Hain Lifescience Nehren, Germany) was used for the hybridization process. All hybridization reagents and solutions: pre-heated hybridization and stringent buffers, diluted conjugate and substrate solutions, rinsing and sterile distilled water were placed into their respective colour-coded slots in the GT-Blot $48^{\circledR}$. Then suction heads were placed into corresponding colour coded solutions. Equal volumes $(20 \mu \mathrm{l})$ of denaturing solution and amplicons were mixed together in individual wells of a tray placed in the GT-Blot $48^{\circledR}$ and allowed to stand for five minutes at $25^{\circ} \mathrm{C}$. During the period of denaturation sample codes were written on the strips and placed into respective sample wells. The automated process of dispensing and aspirating the various solutions was set in a sequential order: (hybridization, stringent wash, rinsing, conjugate, rinsing, sterile distilled water, substrate, sterile distilled water). The strips were then dried using the heating systems in the GT-Blot $48^{\circledR}$.

\subsubsection{Evaluation and Interpretation of Results}

The fully dried strips were scanned using GenoScan ${ }^{\circledR}$ (Hain Lifescience, Nehren, Germany) which generated an automated read-out of the banding patterns. The strips were pasted on evaluation sheets included in the kit. 


\subsection{Data Analysis}

Data analysis was carried out using Surveillance of Drug Resistance Tuberculosis (SDRTB4) Epi-Info based WHO software for entering and analyzing data from anti-TB drug resistance surveys. Prevalence of drug resistance was calculated based on the number of cases with drug susceptibility results available.

\subsection{Ethical Considerations}

This study obtained ethical approval from the Institutional Review Board of NMIMR, University of Ghana, Legon, Accra, Ghana (protocol number: 003/04-05).

\section{Results}

\subsection{Demographic Characteristics of Patients}

A total of 524 presumptive TB patients were included in the study of which 410 eligible patients based on study inclusion and exclusion criteria were selected after interviews were conducted by nurses at various diagnostic centers. Isolates were obtained from 345 patients. Majority 234/345 (68\%) of the patients were males. The median age for all patients was 35.6 years at a range of 11 and 82 years. Three hundred and twenty four (93.9\%) of the isolates were from new cases whiles 21 (6.9\%) were old cases. A third, 125 (36.2\%) of the study patients were from the Greater Accra region (Table 1).

\subsection{Isolation and Identification of Isolates}

Twenty-five cultures were contaminated $(6.1 \%)$ while 40 (9.8\%) showed no growth after eight weeks of incubation at $37^{\circ} \mathrm{C}$ and thus were excluded from further analysis. Three hundred and forty-five $(84.1 \%)$ positive cultures were obtained and identified as members of Mycobacterium tuberculosis complex (MTBC). Further identification of 133 MTBC strains using the GenoType MTBC were as follows: M. tuberculosis, 126 (94.7\%) and M. africanum 7 (5.3\%). Overall, $256 / 345$ (74.2\%) isolates were sensitive to all four drugs tested while $89 / 345$ (25.8\%) showed any form of resistance. Of all resistant isolates, 76/89 (85.4\%) and 13/89 (14.6\%) were from new and old cases respectively. Resistance to one, two, three and four drugs were detected in 56/345 (16.2\%), 27/345 (7.8\%), 4/345 (1.2\%) and 2/345 (0.6\%) isolates respectively. Monoresistance against streptomycin was the highest $41 / 345$ (11.9\%), followed by isoniazid $12 / 345$ (3.5\%) and ethambutol 3/345 (0.9\%); rifampicin monoresistance was not observed. The rate of multi-drug resistance (MDR, defined as resistance to at least isoniazid and rifampicin) was low at 5/345 (1.4\%). Among the poly-resistant (defined as resistance to a combination of two or more drugs excluding MDR) isolates, resistance to a combination of streptomycin and isoniazid occurred frequently (Table 2). Of the 133 isolates whose resistance patterns to isoniazid and rifampicin were compared using both biochemical and molecular DST methods, 127 (95.5\%) gave concordant susceptibility test patterns; 
Table 1. Demographic characteristics of study patients $(n=345)$.

\begin{tabular}{|c|c|c|}
\hline Characteristic & Number & Percentage (\%) \\
\hline \multicolumn{3}{|l|}{$\underline{\text { Sex }}$} \\
\hline Male & 234 & 68 \\
\hline Female & 111 & 32 \\
\hline \multicolumn{3}{|l|}{ Age range (years) } \\
\hline$<15$ & 4 & 1.1 \\
\hline $15-24$ & 52 & 15.1 \\
\hline $25-34$ & 109 & 31.6 \\
\hline $35-44$ & 72 & 20.9 \\
\hline $45-54$ & 60 & 17.4 \\
\hline $55-64$ & 27 & 7.8 \\
\hline $65+$ & 21 & 6.1 \\
\hline \multicolumn{3}{|l|}{ Region of residence $^{\mathrm{a}}$} \\
\hline Ashanti & 30 & 8.7 \\
\hline Brong-Ahafo & 23 & 6.7 \\
\hline Central & 22 & 6.4 \\
\hline Eastern & 54 & 15.7 \\
\hline Greater Accra & 125 & 36.2 \\
\hline Northern & 18 & 5.2 \\
\hline Volta & 20 & 5.8 \\
\hline Western & 22 & 6.4 \\
\hline Upper East & 18 & 5.2 \\
\hline Upper West & 13 & 3.7 \\
\hline \multicolumn{3}{|l|}{$\underline{\text { TB Status }}$} \\
\hline New case ${ }^{\mathrm{b}}$ & 324 & 93.9 \\
\hline Old case ${ }^{c}$ & 21 & 6.1 \\
\hline
\end{tabular}

${ }^{\text {a }}$ Regional breakdown of study patients; ${ }^{\mathrm{b}}$ Pulmonary TB patients in the selected diagnostic centres who have never been treated for $\mathrm{TB}$ or have been treated for a period less than one month. ${ }^{\mathrm{c}}$ All patients who have been treated for TB before for a period more than one month.

121 (95.3\%) were susceptible to both drugs, 6 (4.7\%) were resistant to isoniazid. Six isolates had discordant results from the two DST methods: four were susceptible to both drugs from biochemical DST but were monoresistant to isoniazid according to the molecular DST. On the other hand, two isolates were respectively monoresistant to isoniazid and rifampicin using biochemical DST but were susceptible to both drugs in each case with molecular DST (Table 3).

\section{Discussion}

This is the first nationwide TB drug resistance survey to be conducted in the country since the establishment of the NTP in 1994. That notwithstanding there 
Table 2. Pattern of resistance to first line anti-tuberculosis drugs in Ghana.

\begin{tabular}{|c|c|c|c|c|c|c|}
\hline & \multicolumn{3}{|c|}{ New cases } & \multicolumn{3}{|c|}{ Previously treated cases } \\
\hline & $\mathbf{N}$ & $\%$ & $95 \% \mathrm{CI}$ & $\mathbf{N}$ & $\%$ & $95 \% \mathrm{CI}$ \\
\hline Total no. of cases tested & 324 & 93.9 & $(90.6,96.3)$ & 21 & 6.1 & $(0.3,35.7)$ \\
\hline Sensitive to all 4 drugs & 248 & 96.9 & $(93.9,98.7)$ & 8 & 3.1 & $(0.0,41.3)$ \\
\hline \multicolumn{7}{|l|}{ Any resistance } \\
\hline $\mathrm{H}$ & 33 & 76.7 & $(58.8,89.6)$ & 10 & 23.3 & $(3.7,58.9)$ \\
\hline $\mathrm{R}$ & 2 & 33.3 & $(0.2,96.0)$ & 4 & 66.7 & $(14.5,98.1)$ \\
\hline E & 7 & 77.8 & $(34.5,98.2)$ & 2 & 22.2 & $(0.0,93.1)$ \\
\hline S & 59 & 81.9 & $(69.8,90.7)$ & 13 & 18.1 & $(2.9,48.5)$ \\
\hline \multicolumn{7}{|l|}{$\underline{\text { Monoresistance }}^{\mathrm{a}}$} \\
\hline $\mathrm{H}$ & 12 & 100 & $(73.5,100.0)$ & 0 & - & $(-)$ \\
\hline $\mathrm{R}$ & 0 & - & $(-)$ & 0 & - & $(-)$ \\
\hline $\mathrm{E}$ & 3 & 100 & $(29.2,100.0)$ & 0 & - & $(-)$ \\
\hline S & 38 & 92.7 & $(79.4,98.6)$ & 3 & 7.3 & $(0.0,76.3)$ \\
\hline \multicolumn{7}{|l|}{$\underline{\text { Multi-drug resistance }}^{\mathrm{b}}$} \\
\hline$H+R$ & 0 & - & $(-)$ & 0 & - & $(-)$ \\
\hline $\mathrm{H}+\mathrm{R}+\mathrm{E}$ & 1 & 100 & $(2.5,100.0)$ & 0 & - & $(-)$ \\
\hline $\mathrm{H}+\mathrm{R}+\mathrm{S}$ & 0 & - & $(-)$ & 2 & 100 & $(15.8,100.0)$ \\
\hline $\mathrm{H}+\mathrm{R}+\mathrm{E}+\mathrm{S}$ & 0 & - & $(-)$ & 2 & 100 & $(15.8,100.0)$ \\
\hline \multicolumn{7}{|c|}{$\underline{\text { Other resistance patterns }^{c}}$} \\
\hline $\mathrm{H}+\mathrm{E}$ & 1 & 100 & $(2.5,100.0)$ & 0 & - & $(-)$ \\
\hline $\mathrm{H}+\mathrm{S}$ & 18 & 75 & $(49.4,92.8)$ & 6 & 25 & $(1.9,71.4)$ \\
\hline $\mathrm{H}+\mathrm{E}+\mathrm{S}$ & 1 & 100 & $(2.5,100.0)$ & 0 & - & $(-)$ \\
\hline $\mathrm{R}+\mathrm{E}$ & 0 & - & $(-)$ & 0 & - & $(-)$ \\
\hline $\mathrm{R}+\mathrm{S}$ & 1 & 100 & $(2.5,100.0)$ & 0 & - & $(-)$ \\
\hline$R+E+S$ & 0 & - & $(-)$ & 0 & - & $(-)$ \\
\hline$E+S$ & 1 & 100 & $(2.5,100.0)$ & 0 & - & $(-)$ \\
\hline
\end{tabular}

$\mathrm{H}=$ isoniazid; $\mathrm{R}=$ rifampicin; $\mathrm{S}=$ streptomycin; $\mathrm{E}=$ ethambutol; $\mathrm{N}=$ Number of cases; $\mathrm{CI}$ = Confidence interval; ${ }^{2}$ Resistance to only one of the drugs tested; ${ }^{b}$ Resistance to at least both isoniazid and rifampicin; ${ }^{\mathrm{c}}$ Resistance to a combination of two or more drugs other than multi-drug resistance pattern.

Table 3. Concordance between Phenotypic and Molecular DST patterns.

\begin{tabular}{lcccccc}
\hline & \multicolumn{2}{c}{ Concordant $(\mathrm{n}=127)^{\mathrm{a}}$} & \multicolumn{2}{c}{ Discordant $(\mathrm{n}=6)^{\mathrm{b}}$} & Total \\
\cline { 2 - 5 } & Susceptible & Resistance & Susceptible & Resistance & $(\mathrm{N}=133)$ \\
\hline Phenotypic DST $^{\mathrm{c}}$ & 121 & 6 & 4 & 2 & 133 \\
Molecular DST $^{\mathrm{d}}$ & 121 & 6 & 2 & 4 & 133 \\
Agreement (\%) & 100 & 100 & 50 & 50 & \\
\hline
\end{tabular}

DST $=$ Drug susceptibility test; ${ }^{a}$ Number of isolates that had the same DST patterns for both Phenotypic

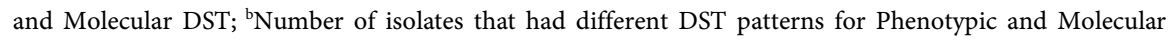
DST; 'DST using biochemical methods; ${ }^{\mathrm{d} D S T}$ using GenoType MTBC assay (Hain Lifescience, Nehren, Germany). 
have been some anti-TB drug resistance studies prior to this survey in Ghana but not on a national scale [5] [6]. This study therefore aimed to identify mycobacterial species causing $\mathrm{TB}$ and to study the pattern of resistance of isolates to common first line anti-TB drugs. Majority of the study patients were males as well as people within the productive age group and this aligns with observations from other studies [11] [12]. The identification of the strains revealed the presence of M. tuberculosis and M. africanum. This result compares with a previous study in Ghana by Yeboah-Manu et al. [13] in which these species were detected. Resistance of members of $M$. tuberculosis complex to anti-TB drugs is largely a man-made problem as wild type (strains that have never been exposed to anti-TB drugs) are almost never clinically resistant; though natural resistance to specific drugs has been documented for M. bovis, i.e. pyrazinamide [14]. The high number of drug susceptible isolates observed in this study corroborates previous reports which indicate that a high performing DOTS programme quite often results in increased rate of treatment success [15]. In this survey, 256/345 (74.2\%) of the isolates studied were susceptible to all the first line drugs used for treatment. The overall rate of resistance $89 / 345$ (25.8\%) was relatively lower than previous studies in Ghana, which were conducted in individual town and district per study [5] [6]. It was emphasized that resistance to one drug only was very high particularly streptomycin. The high resistance to streptomycin may be attributed to selective drug pressure due to its excessive use in treating other bacterial infections and incomplete treatment course [16]. This observation justifies the need to administer multi-drug therapy to minimize resistance to a single drug. High resistance to both isoniazid and streptomycin as seen in our study is a cause for alarm. This is because these drugs are key components in the first line anti-TB drug regimen and have been used in Ghana for a longer period than rifampicin and ethambutol. Primary resistance (an indication of continuous transmission of the disease in the various communities) can be used as a marker for measuring the performance of $\mathrm{TB}$ control programmes as far as effective treatment is concerned. It is worth noting that the overall rate of primary resistance was high $76 / 345$ (22.0\%) in this study compared to similar studies from Uganda (19.8\%) [12], but slightly lower than results from previous studies in Ghana by Owusu-Dabo et al. [16] which reported a rate of 23.5\%. In comparison with results from other African countries: (Sierra Leone, 52.9\%; Kenya, 37.0\%; Zimbabwe, 13.9\%) [17], the $13 / 345$ (3.8\%) rate of acquired resistance observed in our study was relatively low. In this study, all the MDR isolates (1.4\%) were observed to be resistant in addition to one or two other first line drugs. Though this MDR rate may be low compared to what was reported from a study in Cote D'Ivoire (5.3\%) [11], it still reinforces the need for continuous strengthening of the DOTS system to improve treatment. The rising concern of MDR-TB cannot be over emphasized with the prevailing incidence of new TB patients infected with strains that have developed resistance to one or two of the potent drugs prior to treatment. During the period of the conduct of this study there was un- 
availability of second-line anti-TB drugs in Ghana; the only option available to patients who had failed the standard retreatment regimen was more often than not a repeat of the same category II regimen. This unfortunately was recipe for continuous transmission of resistant TB strains to community members and health care workers who provide care for these patients. We note here that two isolates that showed resistance to all four drugs tested were from previously treated cases. This corroborates the generally held view that polyresistant TB are more frequently seen in previously treated cases than new cases [18]. Presence of HIV infection, history of diabetes mellitus, closeness to a known TB patient, previous anti-TB treatment, overcrowding, malnutrition and poverty among others have been hypothesized to be some of the risks factors for TB including drug resistant forms [19]. In this study, our focus was on the pattern of first-line anti-tuberculosis drug resistance and did not go further to do risk factor analysis. Our study had some limitations. The categorization of patients as new or previously treated cases was based upon the information given by the patients and may not have been accurate. Again, drug resistance was tested for only culture positive cases. Due to certain operational challenges during storage, not all the isolates could be retrieved for molecular identification and DST. Furthermore, funds for the molecular testing became available in 2017 hence the delay in the publication of the findings. Although the data are almost a decade old, they do provide a baseline that could be relied on for subsequent nationwide drug resistance survey in Ghana.

\section{Conclusion}

The present survey indicates that $M$. tuberculosis remains the leading MTBC species causing TB in Ghana. On the other hand, there are high levels of resistance to first-line anti-TB drugs especially streptomycin and isoniazid. However, the rate of MDR TB was relatively low at $1.4 \%$. This work reports high resistance involving first line drugs among new cases. It is therefore imperative to aggressively deal with ongoing transmission to complement the gains made in other aspects of control measures.

\section{Acknowledgements}

We are grateful to the regional and district health directorates, regional technologists and staff of all the laboratories visited for sample collection and transportation. We are also grateful to the Global Fund against HIV/AIDS, TB \& Malaria and the Ghana Health Service for their financial support. The molecular part of the study was funded by DELTAS Africa Initiative [Afrique One-ASPIRE/ DEL-15-008]. Afrique One-ASPIRE is funded by a consortium of donors including the African Academy of Sciences (AAS), Alliance for Accelerating Excellence in Science in Africa (AESA), the New Partnership for Africa's Development Planning and Coordinating (NEPAD) Agency, the Wellcome Trust [107753/A/15/Z] and the UK government. 


\section{Competing Interest}

Authors have declared that no competing interests exist.

\section{Authors' Contribution}

KKA came out with the study design and supervised the laboratory work and contributed to the writing and editing of the manuscript. RO, CB and KOD performed the conventional laboratory work. SOA performed the molecular laboratory work, contributed to the manuscript writing and data analysis. GIM assisted with the laboratory analysis and manuscript writing. MJM and DOA contributed to data analysis and editing of the manuscript. FAB contributed to the study implementation by providing reagents and equipment. All authors read and approved the final manuscript before submission.

\section{References}

[1] National Tuberculosis Programme (2001) Tuberculosis Control Strategic Plan for Ghana. Ministry of Health, Accra.

[2] World Bank Data. Incidence of Tuberculosis (per 100,000 People). http://data.worldbank.org/indicator/SH.TBS.INCD?locations $=\mathrm{GH}$

[3] National Tuberculosis Programme (2011) Plan to Initiate and Expand Drug Resistant TB Management in Ghana. Ghana Health Service, Accra.

[4] National Tuberculosis Programme (2003) Annual Tuberculosis Report. Ghana Health Service/Ministry of Health, Ghana.

[5] van der Werf, T.S., Groothuis, D.G. and van Kingeren, B. (1989) High Initial Drug Resistance in Pulmonary Tuberculosis in Ghana. Tubercle, 70, 249-255.

https://doi.org/10.1016/0041-3879(89)90019-6

[6] Lawn, S.D., Frimpong, E.H., Al-Ghusein, H., et al. (2001) Pulmonary Tuberculosis in Kumasi, Ghana: Presentation, Drug Resistance, Molecular Epidemiology and Outcome of Treatment. West African Journal of Medicine, 20, 92-97.

[7] World Health Organization (2003) Guidelines for Surveillance of Drug Resistance in Tuberculosis, WHO/CDS/2003.3. World Health Organization, Geneva.

[8] World Health Organization (2003) Treatment of Tuberculosis Guideline for National Programmes. 3rd Edition, WHO/CDC/TB/2003. 313, World Health Organization, Geneva.

[9] Instructions for Use for GenoType Mycobacterium MTBC.

http://www.hain-lifescience.de/include_datei/kundenmodule/packungsbeilage/dow nload.php?id=981

[10] Instructions for Use for GenoType Mycobacterium MTBDRplus.

http://www.hain-lifescience.de/include_datei/kundenmodule/packungsbeilage/dow nload.php?id=936

[11] Dosso, M., Bonard, D., Msellati, P., et al. (1999) Primary Resistance to Antituberculosis Drugs: A National Survey Conducted in Cote d'Ivoire in 1995-1996. International Journal of Tuberculosis and Lung Disease, 3, 805-809.

[12] Bretzel, G., Aziz, M., Wendl-Richter, U., et al. (1999) Anti Tuberculosis Drug Resistance Surveillance in Uganda 1996-1997. International Journal of Tuberculosis and Lung Disease, 3, 810-815. 
[13] Yeboah-Manu, D., Asante-Poku, A., Bodmer, T., et al. (2011) Genotypic Diversity and Drug Susceptibility Patterns among M. tuberculosis Complex Isolates from South-Western Ghana. PLOS ONE, 6, e21906.

[14] Niemann, S., Richter, E. and Rüsch-Gerdes, S. (2000) Differentiation among Members of the Mycobacterium tuberculosis Complex by Molecular and Biochemical Features: Evidence for Two Pyrazinamide-Susceptible Subtypes of M. bovis. Journal of Clinical Microbiology, 38, 152-157.

[15] Kehinde, A.O., Obaseki, F.A., Ishola, O.C., et al. (2007) Multidrug Resistance to Mycobacterium tuberculosis in a Tertiary Hospital. Journal of National Medical Association, 99, 1185-1189.

[16] Owusu-Dabo, E., Adjei, O., Meyer, C., et al. (2006) Mycobacterium tuberculosis Drug Resistance, Ghana. Emerging Infectious Diseases, 12, 1170-1172. https://doi.org/10.3201/eid1207.051028

[17] World Health Organisation (2007) Anti Tuberculosis Drug Resistance in the World. The WHO/IUATLD Global Project on Anti-Tuberculosis Drug Resistance Surveillance. WHO/TB/97.229, World Health Organization, Geneva.

[18] Mwinga, A. (2001) Drug-Resistant Tuberculosis in Africa. Annals of New York Academy of Science, 953, 106-112.

https://doi.org/10.1111/j.1749-6632.2001.tb11366.x

[19] Diande S., Sangare L., Kouanda S., et al. (2009) Risk Factors for Multidrug-Resistant Tuberculosis in Four Centers in Burkina Faso, West Africa. Microbiology Drug Resistance, 15, 217-221. https://doi.org/10.1089/mdr.2009.0906 


\section{List of Abbreviations}

AFB: Acid fast bacilli

A-LYS: Alcalic lysis

Anti-TB: Anti-tuberculosis

CPC: Cetylpyridinium Chloride

DOTS: Directly Observed Treatment Short course

DST: Drug susceptibility testing

E: Ethambutol

H: Isoniazid

HIV: Human Immunodeficiency Virus

LJ: Lowenstein-Jensen

MDR: Multi-drug resistance

MTBC: Mycobacterium tuberculosis complex

NMIMR: Noguchi Memorial Institute for Medical Research

NTP: National Tuberculosis Control Programme

TB: Tuberculosis

PCR: Polymerase chain reaction

PTB: Pulmonary TB

R: Rifampicin

S: Streptomycin

WHO: World Health Organization

ZN: Ziehl-Neelseen 